\title{
Kinetic Roughening in Deposition with Suppressed Screening
}

\author{
Peter Nielaba $^{a}$ and Vladimir Privman ${ }^{b}$ \\ ${ }^{a}$ Institut für Physik, Universität Mainz, Staudingerweg 7, D-55099 Mainz, Germany \\ ${ }^{b}$ Department of Physics, Clarkson University, Potsdam, New York 13699-5820, USA
}

\begin{abstract}
Models of irreversible surface deposition of $k$-mers on a linear lattice, with screening suppressed by disallowing overhangs blocking large gaps, are studied by extensive Monte Carlo simulations of the temporal and size dependence of the growing interface width. Despite earlier finding that for such models the deposit density tends to increase away from the substrate, our numerical results place them clearly within the standard KPZ universality class.
\end{abstract}

PACS numbers: 68.10.Jy, 82.20.Wt 
The standard, KPZ [1] model of kinetic roughening of growing surfaces (reviewed, e.g., in [2-3]) yields the scaling prediction for the interfacial width $W$ as a function of time, $T$, and substrate size, $L$,

$$
W \simeq L^{\zeta} F\left(T L^{-z}\right)
$$

where for 1D substrates,

$$
\begin{gathered}
\zeta_{\mathrm{KPZ}}=\frac{1}{2}, \\
z_{\mathrm{KPZ}}=\frac{3}{2} .
\end{gathered}
$$

In fact, the value $\zeta=1 / 2$ is typical of $1 \mathrm{D}$ fluctuating interfaces. However, (3) is characteristic of the KPZ universality class. For instance, for stationary fluctuating interfaces, (3) is replaced by $z=2$. These values have been well established by numerical simulations and are believed to be exact (in 1D).

In an interesting study [4], Krug and Meakin argued that to the leading order, the KPZ fluctuations affect the growth rate by introducing, in the average deposit density $\rho(h)$ at the height $h$ away from the substrate, the term

$$
\Delta \rho_{\mathrm{KPZ}} \simeq \lambda h^{-2(1-\zeta) / z} .
$$

This expression applies for times $T$ large enough so that the density has reached its limiting value at $h$, and assuming no finite- $L$ effects, i.e., for infinite substrates. The coefficient $\lambda>0$ is related to the nonlinear growth term is the KPZ theory [1-3]. Specifically, in 1D, this contribution suggests the coverage decreasing to the limiting 
large- $h$ value according to the power law $\Delta \rho \sim h^{-2 / 3}$. The prediction (4) has been verified for several ballistic deposition models in 1D and 2D [4-5].

A recent study [6] of certain 1D models [7] with screening suppressed by disallowing deposition events which block large gaps, yielded a surprising conclusion that in these models the density actually $\underline{\text { increases }}$ away from the substrate according to the power law

$$
\Delta \rho=\rho(h)-\rho(\infty) \simeq-C h^{-\phi}
$$

where $C>0$ and $\phi \simeq 0.3$. An interesting question thus arises: are these models in a universality class different from KPZ? An alternative is that the KPZ contribution to the density, (4), is possibly not seen because the added mechanism of "compactification" due to suppression of screening, elucidated in [6], yields the density term (5) with negative exponent $\phi \underline{\text { smaller }}$ in absolute value than the KPZ-contribution exponent.

In this work we report extensive numerical simulations which clearly place the models under consideration [6-7], to be defined in detail shortly, within the KPZ universality class. In fact, the exponent values and scaling form, (1)-(3), are confirmed quite accurately.

We consider multilayer deposition of $k$-mer "particles" on the linear lattice. The deposition attempts are "ballistic;" particles arrive at a uniform rate per site. The group of those $k$ lattice sites which are targeted in each deposition attempt is examined to find the lowest layer $n \geq 1$ such that all the $k$ sites are empty in that layer (and all layers above it). Note that initially the substrate is empty, in all the sites and layers $1,2,3, \ldots$.. If the targeted group of sites is in the layer $n=1$, then the particle is deposited: the $k$ sites become occupied. However, if the targeted layer is $n>1$, then the deposition attempt is accepted only provided no gaps of size $k$ or larger are thereby covered in 
layer $n-1$. Otherwise the attempt is rejected.

Note that since the layer $n$ is the lowest with all the $k$ sites empty, then in layer $n-1$, one or two $k$-mer particles partially or fully cover this group of $k$ sites. Thus within the $k$-span chosen, the layer $n-1$ can have at most $k-1$ empty sites. Furthermore, there can be at most 1 continuous sequences of empty sites which includes one of the ends of the $k$-span in layer $n-1$. If such a continuous empty-site sequence actually extends outside the $k$-span (i.e., it includes empty sites in layer $n-1$ immediately neighboring the chosen $k$-group of sites), than we disallow deposition if this "external" gap is $k$-site or larger. Figures of illustrative configurations can be found in [6].

Thus we disallow overhangs which would partially or fully block (screen) those gaps which are large enough to accommodate future deposition events (in layers $n-1$ or lower). The final, large-time configuration in each layer contains gaps of at most $k-1$ consecutive empty sites. However, the gaps can be of unlimited size in the direction perpendicular to the substrate (extending from layer to layer). In fact, deposition in lower layers $1, \ldots, N$ is unaffected by deposition in layers $N+1$ on. For layer $n=1$, exact solution for the fraction of occupied sites and for some correlation properties is known [8]; this problem corresponds to monolayer random sequential adsorption in 1D.

We studied the growth of the interfacial width in this deposition process. Specifically, we define $L$ as the number of sites in the lattice (and we use periodic boundary conditions). The Monte Carlo (MC) time variable $T$ is conveniently defined to have one deposition attempt per lattice site per unit time. The heights of the deposit, $h_{j}$, at sites $j=1, \ldots, L$, were defined as the number of layers from the substrate to the last occupied layer, at each lattice site $j$. The r.m.s. width was defined as 


$$
W=\left\langle\sqrt{\frac{1}{L} \sum_{j=1}^{L} h_{j}^{2}-\left(\frac{1}{L} \sum_{j=1}^{L} h_{j}\right)^{2}}\right\rangle
$$

where the average $\langle\ldots\rangle$ over independent MC runs was taken after calculating the square root.

Figure 1 presents results for large substrates, $L=2000$. These data, for $T \leq 200$, were typically averaged over 1000 independent MC runs. There is no visible size effect for $L=2000$. Thus, relation (1) is replaced by

$$
W \simeq T^{\zeta / z} \quad(L \rightarrow \infty)
$$

which corresponds to assuming that the scaling function $F(t)$ behaves according to $\sim t^{\zeta / z}$ for small arguments,

$$
t=T L^{-z}
$$

Least-squares fits of the largest- $T$ data indicate that the exponent in $(7)$ tends to $1 / 3$. For instance, for data in the range $150 \leq T \leq 200$, we get $0.383,0.345,0.316$, $0.304,0.324$, for $k=2,3,5,8,10$, respectively. Based on our analyses, we propose the estimate

$$
\zeta / z=0.34 \pm 0.04
$$

clearly excluding values such as $1 / 2$ or $1 / 4$ and favoring the KPZ [1-3] prediction $1 / 3$. We also checked this estimate for several data sets taken at $L=1000$ and 1500 . The results were unchanged. For larger $k$, e.g., 10, the onset of the finite- $L$ saturation can be seen for $L=\mathrm{O}(1000)$. 
Analysis of the finite- $L$ properties was complicated by two facts. Firstly, to see finite- $L$ saturation, simulations had to be done for large times. Secondly, we found that the statistical noise in the data became significant at saturation. Thus averages over many independent runs were required. We restricted our extensive MC runs to one $k$ value, $k=3$. This value was favored because generally, other conditions being equal, the observed statistical noise became larger as $k$ increased. On the other hand, the $k=3$ large- $L$ data in Figure 1 yield exponent closer to $1 / 3$ than the $k=2$ data, suggesting, possibly, smaller corrections to the leading scaling behavior.

Figure 2 shows well saturated data for $k=3$ and lattice sizes $L=20,40,60$, 80, 100. These were averaged over typically 10000 MC runs. Shown are also data for $L=300$, averaged over $2000 \mathrm{MC}$ runs, which have not attained saturation for the largest times reached in the simulation. The $L=2000$ data from Figure 1 are also included for comparison.

The spread of the saturation values at larger $T$ selected to have only the statistical noise, is shown for $L=20,40,60,80,100$ in Figure 3. For large times, one assumes $F(t \rightarrow \infty) \simeq$ constant in $(1)$ so that the width behaves according to $L^{1 / 2}$. From leastsquares fits to various data subsets for $L=40,60,80,100$, i.e., excluding the data for $L=20$ which seem to be too small to reach the true asymptotic behavior, we propose the estimate

$$
\zeta=0.49 \pm 0.03
$$

As mentioned earlier, this exponent is the same for various 1D universality classes and it cannot be used to identify the KPZ behavior. However, accurate verification of the value $1 / 2$ suggests that our data are generally well within the asymptotic regime for lattice sizes above $\mathrm{O}(40)$. 
Thus, we also attempted the full scaling data collapse, i.e., we checked that the quantity

$$
w=W L^{-1 / 2},
$$

when plotted as a function of $t$ defined in (8), is represented by a unique function $F(t)$; see (1). Of course the data collapse is exact only in the limit $L \rightarrow \infty$ and $T \rightarrow \infty$, with fixed $t$. Figure 4 illustrates the "collapse" for $k=3$, where we used data for $L=80$, 100, 300, 2000, described earlier. We also included data for $L=1000$ which, together with the $L=2000$ data, yield the dense portion of the plot for $w \lesssim 0.3$ (see Figure 4).

All the general expectations on the form of the scaling function $F(t)$ are qualitatively confirmed by our data. There are several extensive numerical studies of the KPZ and other growth-universality classes by scaling data collapse and tests of universality of quantities derived from scaling functions similar to $F(t)$; see, for instance, [9-11]. The quality of our data is comparable to other accurate verifications of the scaling predictions in $1 \mathrm{D}$, though we found no results in the literature to allow direct comparison with the scaling-function data such as Figure 4.

In summary, we found by extensive MC simulations measuring directly the growing interface width, that the models with suppressed screening which show unusual density variation [6] are, in fact, described quite accurately by the KPZ scaling form [1-3] typical of growing interfaces, with the appropriate 1D exponent values.

This work was supported in part by the DFG-Sonderforschungsbereich 262. One of the authors (P.N.) wishes to thank the DFG for Heisenberg fellowship. 


\section{REFERENCES}

[1] M. Kardar, G. Parisi and Y.C. Zhang, Phys. Rev. Lett. 56, 889 (1986).

[2] J. Krug and H. Spohn, in Solids Far from Equilibrium: Growth, Morphology, Defects, edited by C. Godrèche (Cambridge University Press, Cambridge, 1991).

[3] Dynamics of Fractal Surfaces, edited by F. Family and T. Vicsek (World Scientific, Singapore, 1991).

[4] J. Krug and P. Meakin, J. Phys. A90, L987 (1990).

[5] B.D. Lubachevsky, V. Privman and S.C. Roy, Phys. Rev. E47, 48 (1993).

[6] P. Nielaba and V. Privman, Phys. Rev. A45, 6099 (1992).

[7] M.C. Bartelt and V. Privman, J. Chem. Phys. 93, 6820 (1990).

[8] J.J. Gonzalez, P.C. Hemmer and J.S. Høye, Chem. Phys. 3, 228 (1974).

[9] J. Krug, P. Meakin and T. Halpin-Healy, Phys. Rev. A45, 638 (1992).

[10] M. Schroeder, M. Siegert, D.E. Wolf, J.D. Shore and M. Plischke, Europhys. Lett. 24, $563(1993)$.

[11] M. Siegert and M. Plischke, J. Physique I3, 1371 (1993). 


\section{FIGURE CAPTIONS}

Fig. 1. Data for $k=2,3,5,8,10$ on substrates of size $L=2000$. For small $T, W_{k=2}<$ $W_{k=3}<\ldots<W_{k=10}$. For large $T$, the relation is reversed on the average, although the differences, especially for $k=8$ and 10 , are small and fluctuate in sign due to statistical noise. Solid line illustrates slope $1 / 3$.

Fig. 2. Data for $k=3$ on substrates of sizes $L=20,40,60,80,100,300,2000$. For fixed $T, W(L)$-values monotonically increase with $L$. Solid line illustrates slope $1 / 3$.

Fig. 3. Large- $T$ data for $k=3$ on substrates of sizes $L=20,40,60,80$, 100, illustrating the $L$-dependence of the saturation values (with the statistical noise). Solid line illustrates slope $1 / 2$.

Fig. 4. Scaling data collapse according to equation (1), for $k=3$. The scaled width $w$ is plotted as a function of the scaled time $t$, for substrate sizes $L=80,100,300$, 1000, 2000. 